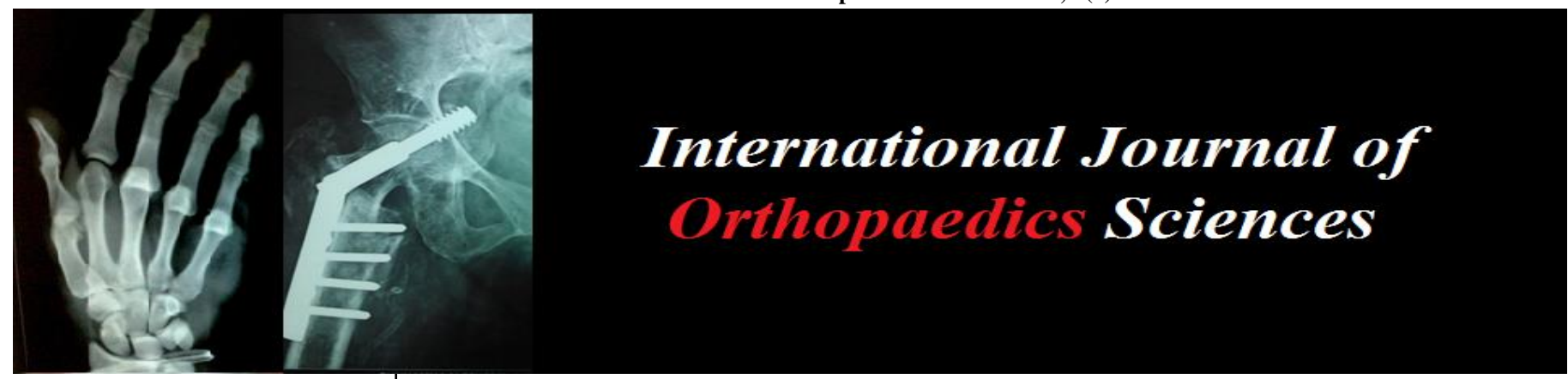

E-ISSN: 2395-1958

P-ISSN: 2706-6630

IJOS 2019; 5(4): 306-309

(C) 2019 IJOS

www.orthopaper.com

Received: 13-08-2019

Accepted: 15-09-2019

Sivakumar M

Assistant Professor, Sivagangai

Medical College, Sivagangai,

Tamil Nadu, India

Sudharsan A

Assistant Professor, Sivagangai

Medical College, Sivagangai,

Tamil Nadu, India

\section{Short term outcome analysis of surgical release for deQuervain's disease: A case series study}

\section{Sivakumar M and Sudharsan A}

DOI: $\underline{\text { https://doi.org/10.22271/ortho.2019.v5.i4f.1690 }}$

\section{Abstract}

Introduction: Though there are various modalities of conservative and minimally invasive management they do not resolve or regress the fibrotic process of the tendon sheath in deQuervain's disease hence surgical release of the tendons from the sheath improves the patients symptoms by correcting the underlying pathology.

Aim: Analysis of functional outcome of surgical release for deQuervain's disease.

Materials and Methods: Prospective evaluation of 20 cases with at least 3 months of unsuccessful conservative therapy undergone open surgical release through longitudinal incision of tendon sheath along with removal of thin strip of extensor retinacilam.

Results: Follow up analysis of the patients were made using DASH scoring and VAS scale and clinical evaluation by negative finkelstein test and range of movements of thumb and wrist. The mean pre operative DASH score is 78.25 where as mean post operative DASH score is 8.03 which is statistically significant. The mean pre operative VAS score is 8.65 where as post operative VAS score is 0.35 also statistically significant.

Conclusion: Surgery is the only treatment for resistant cases after 6 months of conservative measures, and should be carried out as soon as possible. In our study group follow up is short term even though it shows significant outcome.

Keywords: deQuervain's disease, extensor retinaculam, DASH score, VAS

\section{Introduction}

Disability caused by de Quervain disease is highly debilitating leading to restriction of daily activities. Bunnell ${ }^{[1]}$ comments, "A hand without a thumb is no more than a hook." In a classical case of de Quervain disease functional limitations so much as rendering thumb functionless. In recent days, Dequervain's disease is found to represent thickening of tendon sheath [2] rather than tendinitis owing to lack of histopathological evidence of any inflammation in specimens. The fibrosis of involved tendon sheath may be progressive or stationary. By means of conservative management the fibrotic process never regress or resolve. But continued motion of the Tendons within stenosed sheath further aggravates this condition by progressive proliferation of fibrous tissue and scarring. Though there are various modalities of conservative and minimally invasive management as physiotherapy, splinting and corticosteroid injections they do not resolve or regress the fibrotic process of the tendon sheath which may be progressive. Thus surgical release of the tendons from the sheath is the only way of correcting the underlying pathology and prevent the progression, relieving the patients from the symptoms. In our study tendon sheath release along with removal of thin strip of extensor retinaculam gives encouraging results in this condition that has failed traditional nonsurgical modalities.

\section{Materials and Methods}

In this study of prospective evaluation of 20 patients with age range of 20 to 60 years with good general condition who undergone open surgical release of tendon sheath along with removal of thin strip of extensor retinacilam. The study participants were recruited between September 2014 to September 2016 from the Department of Orthopaedics and Traumatology, Madurai Medical College Hospital and Sivagangai Medical College Hospital, Tamil Nadu, 
India. All the patients selected for study were examined according to protocol and clinical and lab investigations carried out in order to get fitness for surgery. Consent of the patient taken for surgery.

\section{Inclusion Criteria}

- At least 3 months of unsuccessful conservative therapy

- Pain interfering with daily activities of living

- Patient age from 20 to 60 years and both sexes

\section{Exclusion Criteria:}

- Comorbid conditions not permitting major surgical procedures

- Patient not willing for surgery

- Pregnancy

- Local arthritis

- Previous surgeries

- Hypothyroidism

- Rheumatoid Arthritis.

Patients underwent a pre-operative evaluation including the following parameters: $\mathrm{Hb}$, blood sugar, Renal function test. All the cases underwent reginal anaesthesia. Intra operative data recorded in the profoma. Wrist and thumb splint was applied. After suture removal on twelfth postop day patients were discharged and adviced to do mild household activities at 3rd week postoperatively gentle scar massage thrice a day, encouraged to do weightlifting around $5 \mathrm{kgs}$, allowed to do cooking and lifting children and at the end of third week stretching exercises of the thumb and wrist followed by gradual strengthening exercises at 4-6 weeks were given. Patients were subjected to regular follow up once in 3 months.
All the patients have been clinically assessed by DASH scoring and VAS scoring system at 3 months interval. Data collected at the end of the study was statistically analyzed.

\section{Surgical procedure}

- Under local anaesthesia with pneumatic tourniquet control patient in supine position, involved limb was kept over the forearm table. Thorough exsanguination was done using Esmarch rubber bandage for better visualization of constricted tendon sheath before inflating the tourniquet.

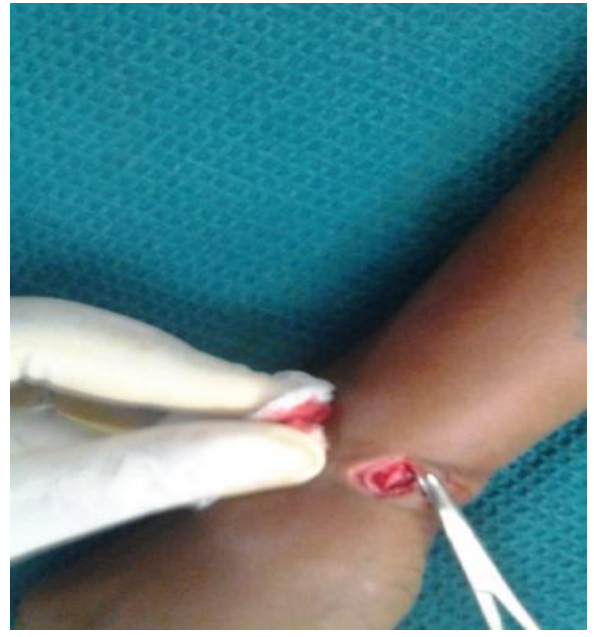

Fig 1: Incision was made over the anterior border of the anatomical snuff box centring the radial styloid in a longitudinal fashion to prevent damage to sensory branches of radial nerve.
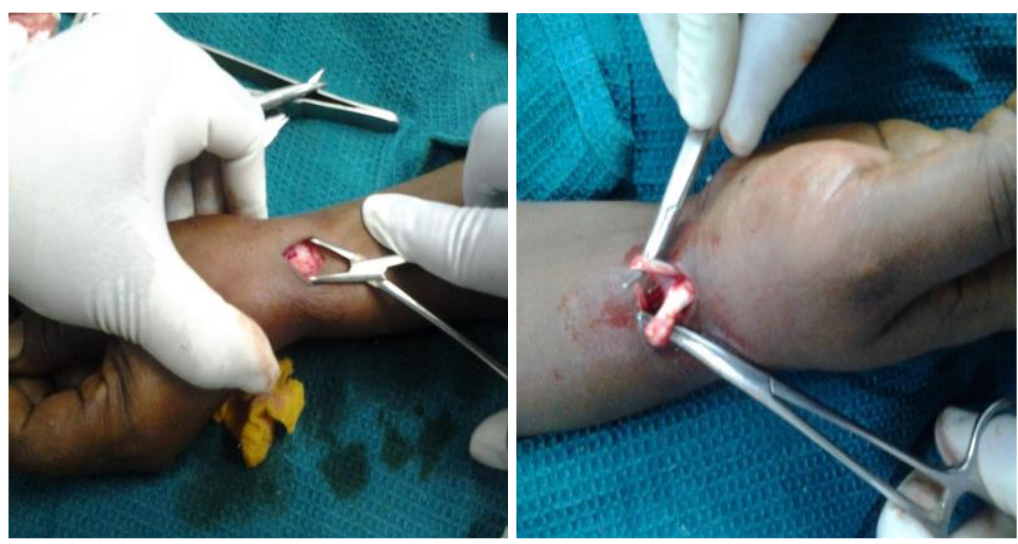

Fig 2: Subcutaneous tissue was exposed after identifying the constricted tendon sheath, incision was made on the tendon sheaths of abductor polliciclongus and extensor pollicisbrevis.

About 2 to $3 \mathrm{~mm}$ strip of dorsally based flap of extensor retinaculum was excised. Wound closed in layers and sterile dressing was applied. Wrist and thumb splint was given for healing of tissues.

\section{Results}

All the twenty patients in our study were treated with surgical release of tendon sheath of abductor pollicieslongus and extensor pollicisbrevis along with excision of thin strip of dorsally based extensor retinaculam. The longest follow up period was 24 months and shortest follow up period was 10 months, mean follow up period was 18 months. Follow up analysis was made using DASH scoring and VAS scale and clinical evaluation by negative finkelstein test ${ }^{7}$ and range og movements of thumb and wrist. Mean age group in all operated cases 28- 45 years among 20 patients 19 were females 1 is male. Involvement of right wrist is 13 and left wrist is 7. Dominant extremity is commonly involved. $75 \%$ patients are housewives performing repetitive forearm wrist activities. The mean pre operative DASH score is 78.25 where as mean post operative DASH score is 8.03 which is statistically significant. The mean pre operative VAS score is 8.65 where as post operative VAS score is 0.35 which is statistically significant. The successful treatment of dequervains disease is defined as more than a $25 \%$ reduction in disabilities of arm shoulder hand (DASH) and visual analog scale without a reintervention after 1 year. Pain relief was achieved in all 20 patient. 2 cases had transient numbness of thumb and no cases show post operative infection. In one of our patients we found an aberrant tendon of abductor pollicislongus which was confirmed by its insertion at the trapezium. The aberrant tendon was identified and released. 


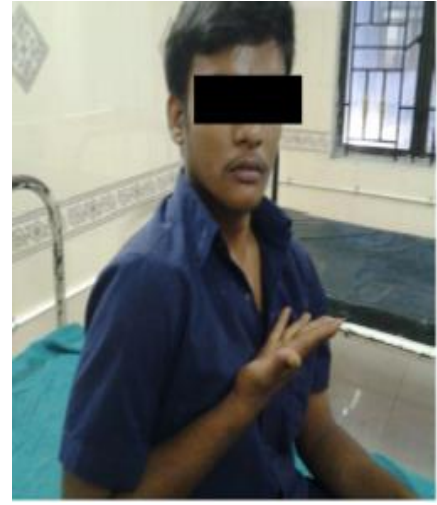

Preoperative

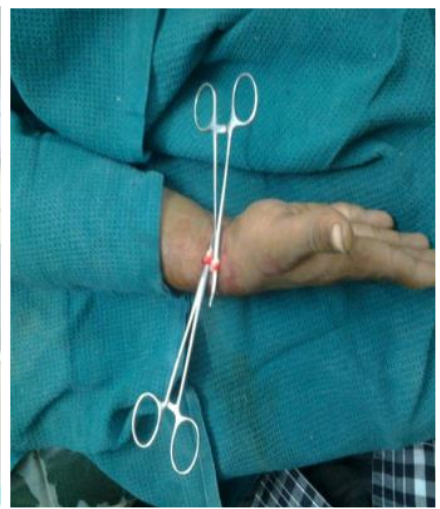

Intraoperative
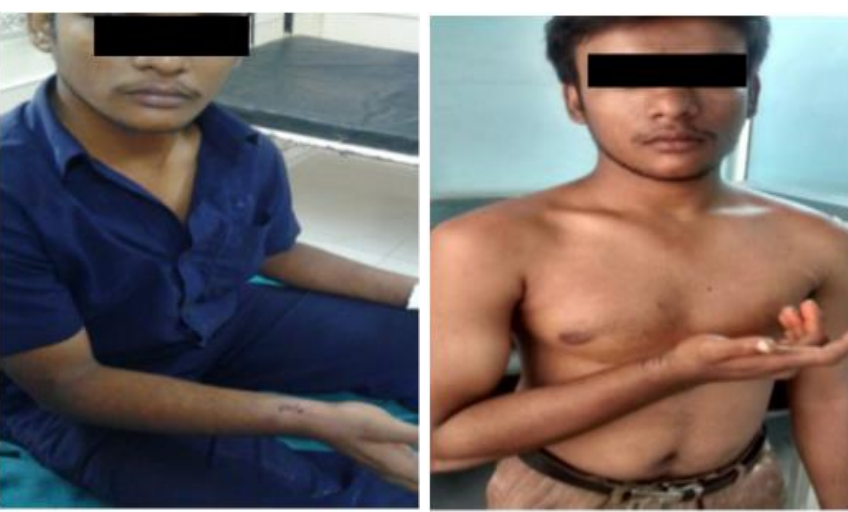

Postoperative

Case 1

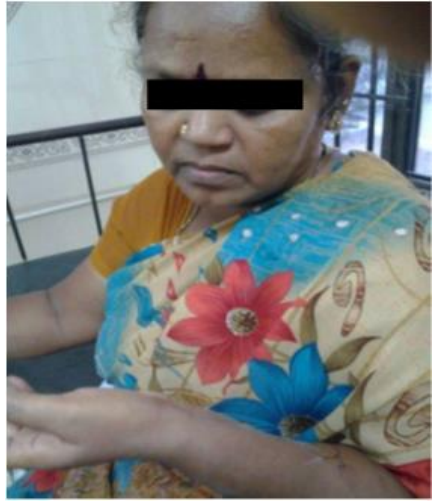

Preoperative

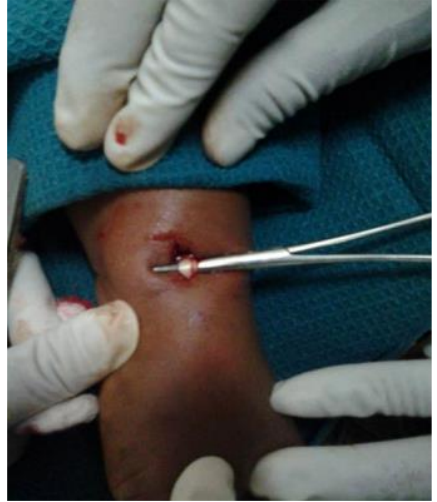

Intraoperative

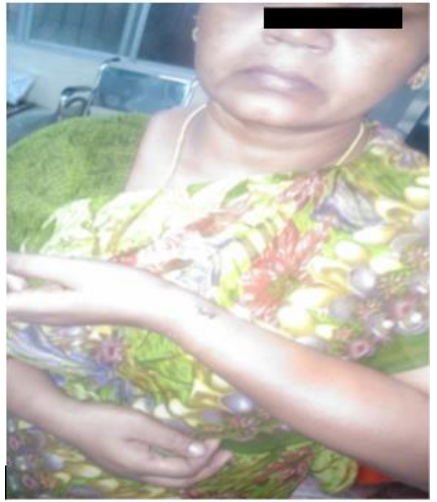

Postoperative

Case 2

Table 1: Clinical variables

\begin{tabular}{|c|c|c|}
\hline Variables & Mean \pm SD & Median (Range) \\
\hline Age (Years) & $35.45 \pm 7.95$ & $35(26)$ \\
\hline Gender (M:F) & $01: 19$ & \\
\hline Side (Right: Left) & $13: 7$ & \\
\hline
\end{tabular}

Table 2: Pre-Op Dash to Post Op Dash

\begin{tabular}{|c|c|c|c|}
\hline & Mean & SD & 'P' Value \\
\hline Pre op & 78.25 & 4.149 & \multirow{2}{*}{$<0.001$} \\
\hline Post op & 8.08 & 1.818 & \\
\hline
\end{tabular}

Table 3: Pre Vas to Post Vas

\begin{tabular}{|c|c|c|c|}
\hline & Mean & SD & 'P' Value \\
\hline Pre op & 8.65 & 0.49 & \multirow{2}{*}{$<0.001$} \\
\hline Post op & 0.35 & 0.49 & \\
\hline
\end{tabular}

Table 4: Complication

\begin{tabular}{|c|c|c|}
\hline Complication & No. of cases & Percentage \\
\hline Numbness & 2 & 10 \\
\hline Nil & 18 & 90 \\
\hline
\end{tabular}

\section{Discussion}

Dequervain's disease is due to chronic repetitive activities which leads to tendon sheath inflammation, fibrosis and stenosis which causes limitation of the wrist and thumb movement. Histopathological evidences reveal congestion, edema, inflammation, fibrosis, thickening of the tendon sheath due to infiltration and proliferation of the round cells and fibrous tissues of the tendon sheath. Surgical release of tendon sheath of abductor pollicislongus and extensor pollicisbrevis along with excision of thin strip of extensor retinaculum which prevents recurrence.

According to S J Mellor and B Ferris ${ }^{[3]}$ longidutinal incision was associated with complications like inadequate decompression in one case $(10 \%)$, six $(60 \%)$ were related to injury to the superficial branch of the radial nerve, two $(20 \%)$ were related to wound infections and one $(10 \%)$ was related to reflex sympathetic dystrophy. Bouras et al. ${ }^{[4]}$ noted nonaesthetic scars in three of 20 patients. Harvey et al. ${ }^{[5]}$ reported six complications in 20 wrists treated surgically, two complications were related to scars, one was a minor wound infection and three were related to a temporary disturbance of the nerve. We encountered nil neurological complication except two cases of numbness over the thumb of the operated limb which resolved with follow up and gives satisfactory clinical results with mean preoperative DASH score of 78.25 and mean postoperative DASH score of 8.08 also mean preoperative VAS score of 8.65 and mean postoperative VAS score of .035 , suggest recommending one-quarter partial removal of the extensor retinaculum on the dorsal side of the wrist in order to prevent problems such as an incomplete release, re-adhesion of the extensor retinaculum and volar subluxation of the tendons after operative treatment of de Quervain's disease. Our study results are comparable with that of Mellor and Ferris study. Further prospective, randomized comparative studies with larger populations are needed. H. J. Kang, I. H. Koh, J. W. Jang, Y. R. Choi et al. ${ }^{[6]}$ study showed the significantly lower rate of nerve injury and greater scar satisfaction in the endoscopic group are consistent with the findings of our study provided all radial nerve symptoms resolved with follow-up regardless of the methods of operation, endoscopic or open release of the first extensor compartment. 
Recently published long term follow up studies of both arthroscopic and open methods of surgical release have demonstrated similar outcome. It appears that either surgical technique is acceptable as long as the pathological tissue is accurately identified and adequately resected, although there are advantages and disadvantages to each procedure, no technique appears superior to others.

Based on our observation short term follow up shows excellent outcome, longer term follow up evaluation are required to substantiate our results. Our study of tendon sheath release along with removal of thin strip of extensor retinaculum gave encouraging results in dequervain's disease that has failed traditional nonsurgical modalities.

\section{Conclusion}

Release of abductor pollicislongus and extensor pollicisbrevis tendon sheath along with thin strip of dorsally based flap of retinaculam removal will improve the functional outcome. DeQuervain's Disease, although frequently unrecognized, is a crippling condition which is easily remedied by surgery. There has been demonstrated a technique which is simple to follow and avoids injury of the cutaneous branch of the radial nerve. The condition is far more prone to occur in women, and this pathologic condition should be considered in the differential diagnosis of persistent pain at the base of the thumb in the region of the radial styloid. Surgery is the only treatment for resistant cases after 6 months of conservative measures, and should be carried out as soon as possible. In this study of short term follow up shows significant outcome.

\section{References}

1. Bunnell, Sterling: Surgery of the Hand. The J. B. Lippincott Co., Philadelphia, 2004, 8.

2. Haggart GE, Winter EF. De Quervain's disease; stenosing tendovaginitis over the radial styloid. Surg Clin North Am. 1948; 28:817-20.

3. Mellor SJ, Ferris BD. Complications of a simple procedure: de Quervain's disease revisited. Int J Clin Pract. 2000; 54(2):76-7.

4. Bouras Y, El Andaloussi Y, Zaouari T, Touil N, Fnini S, Chikhaoui N et al. Surgical treatment in De Quervain's tenosynovitis. Ann Chir Plast Esthet. 2010; 55:42-5.

5. Harvey FJ, Harvey PM, Horsey MW. De Quervain's disease: Surgical or nonsurgical treatment. J Hand Surg Am 1990; 15:83-7.

6. Kang HJ, Koh IH, Jang JW, Choi YR. Endoscopic versus open release in patients with de Quervain's tenosynovitis: a randomised trial. Bone Joint J. 2013; 95-B(7):947-51.

7. Elliott BG. Finkelstein's test: a descriptive error that can produce a false positive. J Hand Surg (Br). 1992; $17: 481-483$. 